\title{
Dietary intake of children with inflammatory bowel disease and of healthy controls
}

\section{T. Chatton ${ }^{1}$, P.M. Marques-Vidal ${ }^{2}$, A. Nydegger ${ }^{1}$}

${ }^{1}$ Pediatric Gastroenterology Hepatology and Nutrition, ${ }^{2}$ Institute of social and Preventive Medicine, Lausanne, Switzerland

\section{Introduction}

Deficiency of energy intake in children is rare in the "developed" countries, but present in many chronic diseases, especially during inflammatory bowel disease (IBD) (Masson, 2006) along with other nutritional deficiencies. This is a major concern in pediatrics, because malnutrition this can have a negative impact on growth and pubertal development.
The assessment of dietary intake by examination is a tool that allows to know the habits and detect certain nutritional deficiencies. It can also calculate energy intake ingested. Thus, it is an integral part of comprehensive care to children with IBD.

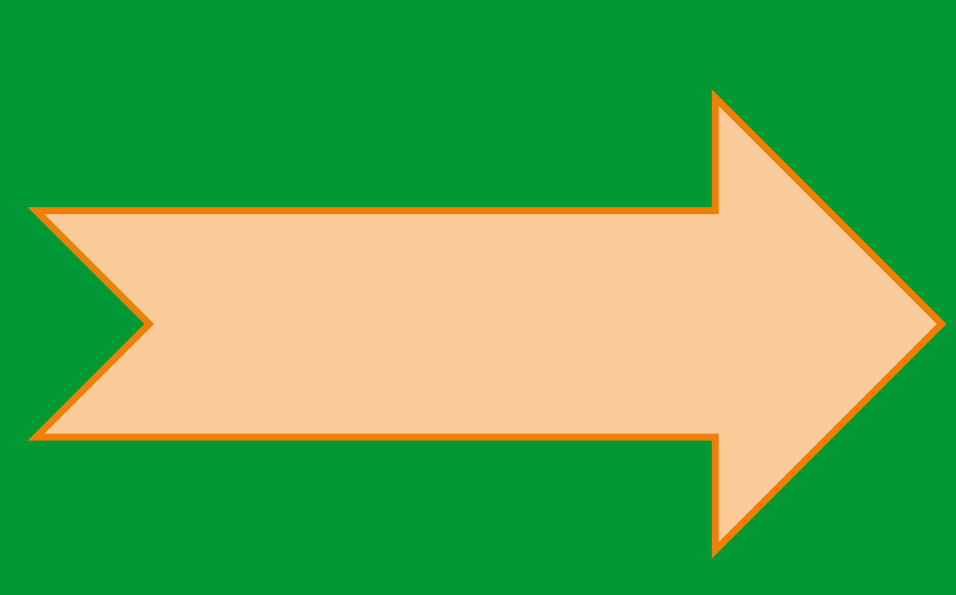

\section{Objective}

Compare food intake among children suffering from a chronic disease of the intestine and healthy children.

\section{Method}

This prospective observational study was conducted in Pediatric Gastroenterology Unit (CHUV, Lausanne, Switzerland) over a period of 14 months (August 2011 - October 2012). It fits in another study on energy metabolism in pediatric patients with IBD.

Dietary intake was assessed by a recall of 24 hours (Fig. 1), and recognized tool widely used in dietary surveys (Willet, 1998). The quantities have been estimated using a document "picture" of food (Fig. 2) and in the memories of children sometimes assisted by their parents. The conversion of food into calories and nutrients has been carried out with the 5.3 software Prodi Expert.

Fig. 1

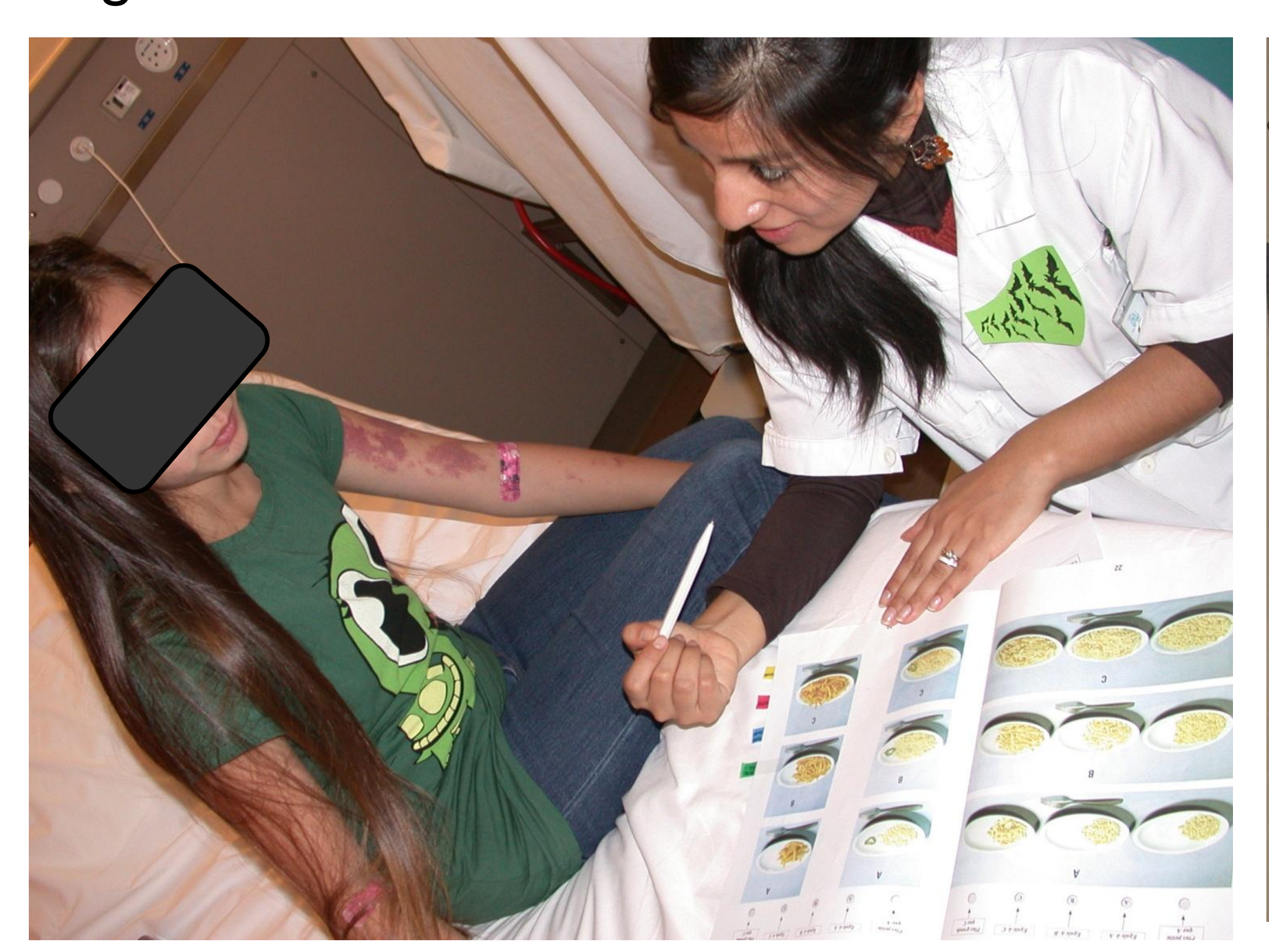

Fig. 2

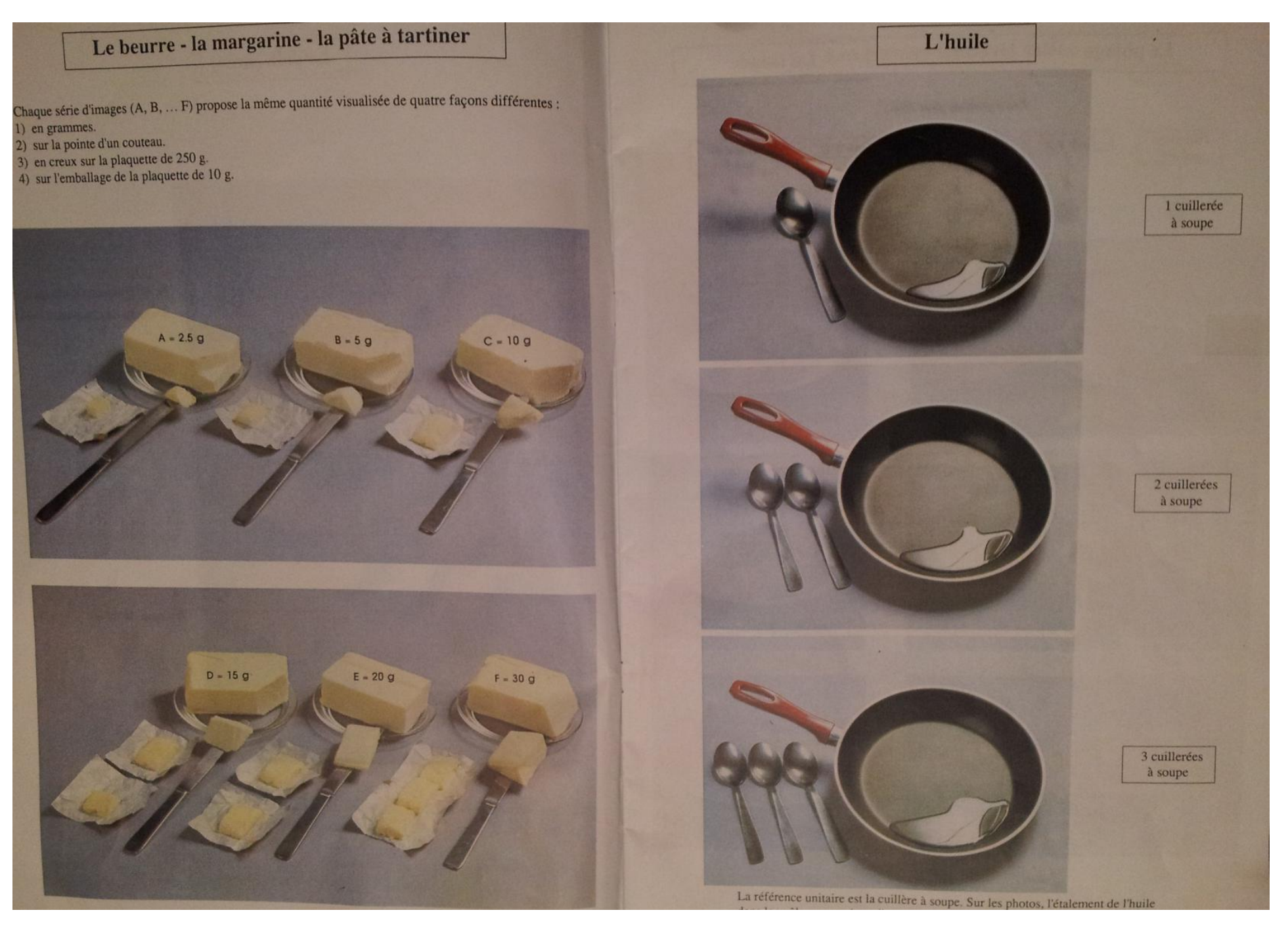

\section{Results}

No significant difference in food consumption was observed between children with IBD and controls (Table 2).

Table 2 :

\begin{tabular}{lccc:c}
\hline Daily intake & Controls & IBD & p-value & ANC \\
\hline Energy consumption (kcal) & $1855 \pm 76$ & $1782 \pm 95$ & 0.59 & + \\
Proteins (\%energy) & $17.7 \pm 1.0$ & $15.6 \pm 1.2$ & 0.23 & $10^{++}$ \\
Lipids (\%energy) & $31.3 \pm 1.6$ & $28.8 \pm 2.0$ & 0.36 & $30^{++}$ \\
Carbohydrates (\%energy) & $49.8 \pm 1.8$ & $54.3 \pm 2.2$ & 0.16 & $50-60^{++}$ \\
Fiber (g) & $17 \pm 1$ & $16 \pm 1$ & 0.45 & $20-30^{++}$ \\
Calcium (mg) & $641 \pm 62$ & $742 \pm 77$ & 0.35 & $1200^{++}$ \\
Iron (mg) & $11.1 \pm 1$ & $9 \pm 1$ & 0.10 & $10-16^{++}$ \\
Magnesium (mg) & $259 \pm 15$ & $258 \pm 19$ & 0.96 & $280-410^{++}$
\end{tabular}

All results are expressed as mean \pm standard deviation. Comparisons were performed by analysis of variance, adjusting for age, sex and micronutrients as the total energy.

t: The energy depends on several factors (age, sex, physical activity).

++ : These values must be adjusted according to age, sex and weight of the child.

In comparison with the Recommended Dietary (Martin, 2001, Table 2 ), we observed in both groups a high protein intake and deficiencies in certain nutrients (fiber, calcium, magnesium).

Note that these results do not apply to a qualitative analysis. It is particularly stood at this level that these two groups have snacks between meals and fat distribution incorrect focusing saturated fats.

\section{Patients}

29 healthy children (controls) and 21 children with IBD (cases - 15 Crohn's disease and ulcerative colitis hemorrhagic 6) were compared (Table 1).

Table 1 :

\begin{tabular}{|ccc|}
\hline & $\begin{array}{c}\text { Controls } \\
(\mathbf{n = 2 9 )}\end{array}$ & $\begin{array}{c}\text { IBD } \\
(\mathbf{n = 2 1 )}\end{array}$ \\
\cline { 2 - 3 } Age (years) & $12.7 \pm 1.9$ & $14.3 \pm 1.3$ \\
Female $(\%)$ & $12(41.4)$ & $11(52.4)$ \\
Male $(\%)$ & $17(58.6)$ & $10(47.6)$ \\
\hline All results are expressed as mean \pm standard deviation.
\end{tabular}

\section{Limitations}

It is considered that the average age of the patients was higher and the accuracy of the $24 \mathrm{~h}$ recall depends on the accuracy of reporting of the topic and the database nutrients.

\section{Conclusion}

No statistical difference was found between children with IBD and controls. However, both groups have nutritional imbalances.

The challenges of a balanced diet in children are multiple and the dietary survey remains an indispensable tool especially in a population at high nutritional risk. 